\title{
Numerical Simulation of a Thermoacoustic Refrigerator
}

\author{
Aniruddha Worlikar and Omar M. Knio \\ Department of Mechanical Engineering \\ The Johns Hopkins University \\ Baltimore, MD 21218, USA
}

\author{
Rupert Klein \\ Fachbereich Sicherheitstechnik \\ Bergische Universität, Gauß-Str. 20 \\ D-42097 Wuppertal, Germany
}

\begin{abstract}
A thermoacoustic device consists of two main components: (1) a resonance tube where the flow is characterized by length scales of the order of the acoustic wavelength, and (2) a stack of plates which are separated by distances much smaller than the acoustic wavelength. This effort focuses on the development of numerical schemes which overcome this scale disparity in an efficient manner. Two approaches are discussed in the paper. The first is a multiple-pressurevariable approach that is suitable for the simulation of resonance tube acoustics and for analyzing interactions between heat addition and long waves. The second is a multi-dimensional model of the stack region which is based on fast solution of the zero-Mach-number conservation equations.
\end{abstract}

\section{Introduction}

The broad objective of this work is to develop computational tools which are useful in the design and analysis of thermoacoustic devices. The basic principle which governs the operation of these devices is the so-called "thermoacoustic effect" [8], which results from the interaction between acoustic waves and thermally-stratified solid surfaces. Thermoacoustic devices harness this effect in order to produce a useful thermodynamic cycle: Thermoacoustic engines convert heat to sound energy, and thus to work. Thermoacoustic refrigerators convert acoustic energy into thermal gradients which enable energy exchange between hot and cold reservoirs. One of the motivations behind the study of the latter is that they eliminate the need for CFC's and, consequently, the associated environmental hazards.

While the soundness of the principle governing the use of thermoacoustic refrigerators has been demonstrated in a number of experimental systems, e.g. [1-3], the thermodynamic efficiency of current prototypes is not competitive yet. Thus, the goal of this work is to construct and implement physical and numerical models which allow us to accurately simulate the flow field within such systems, to analyze their performance and to suggest viable improvements. The difficulties involved in such numerical simulations are considerable. In order for us to discuss them, and to devise suitable approaches, a simplified thermoacoustic refrigeration system is first described.

\section{Simplified thermoacoustic refrigeration system}

The essential components of a thermoacoustic refrigeration system include a resonance tube, a stack of closely-spaced plates, a sound generating device e.g., a loudspeaker, and heat exchangers (Fig. 1). Assume that a standing acoustic wave, with wavelength equal to twice the tube length, has been excited, and consider the motion of fluid particles within the tube. Due to velocity perturbations 
induced by the pressure wave, a particle will be displaced in an oscillatory fashion. Meanwhile, the particle will also undergo pressure and temperature fluctuations. For example, a particle near the right end of the tube is moved towards the right end as the pressure maximum is achievd, while the gas expansion upon pressure drop in the opposite phase of the cycle moves the particle to the left when its temperature drops slightly. Thus, fluid particles generally trace a local temperature gradient by this oscillatory motion. (See Fig. 2 for a sketch.)

The phenomenon described above may be immediately applied to particles in the neighborhood of stack plates. We consider that the stack has thin, closely spaced plates; thus axial conduction along the plates is ignored, but heat exchange between the gas and the plates is retained. Initially, the plates are in thermal equilibrium with the gas. Now, consider a mass particle located between two neighboring plates, as sketched in Fig. 2. As it is moved to the right being heated by compression, it gets hotter than the plate locally and it gives heat to the plate. As it moves to the left and expands, it becomes colder than the plate and receives heat. The net effect of one oscillatory cycle is to move heat along the plate. Over many cycles, unless the plate is heated, its left end and the surrounding gas are cooled; ultimately, the plates will acquire a temperature distribution which at every point has a gradient which is similar to that traced by the particles during their acoustic motion. If heat is added to the left end at some temperature lower than ambient, but higher than the plate's long time equilibrium temperature, a persistent heat transport from this low temperature reservoir to the ambient is established.

\section{Computational difficulties and approach}

There are several challenges involved in numerically simulating the flow field involved: First, we are facing multi-dimensional low-Mach-number vortical flow around the stack. The key physics we are interested in involves heat exchange with the boundary due to small amplitude temperature fluctuations, which in turn are due to the acoustic compression. Hence we need to solve at the leading order, the zero Mach number flow equations [6] including global compression and heat flow.

The stack flow as well as the heat exchange are driven by long-wave acoustics in the tube. Since we have to consider a large number of oscillations in order to reach "steady-state" conditions, there is sufficient time for the acoustics to accumulate weakly nonlinear effects. In addition, the cooling near the plates as well as the acoustic driver will induce large-amplitude temperature and density variations of the gas. Hence, the outer acoustic flow, even though it may be considered quasi-one-dimensional, is by no means trivial to simulate: One needs the capability of computing, efficiently, weakly nonlinear acoustics in a non-homentropic gas with large density variations.

Here, we present two specialized numerical tools that are capable of accurately and efficiently deal with the "inner" (stack flow) and the "outer" (acoustic) problems. At this stage we are able to demonstrate that these tools are in fact adequate to handle the abovementioned difficulties separately and we show first simplified simulations that capture the essential features of thermoacoustic phenomena. We plan to follow two separate routes in future implementations. One route involves a direct coupling of these two codes in a joint computation through an appropriate interface. This will provide a detailed description of the entire flow field, yet may prove too costly to be used in an extensive analysis or in a design study. The second route therefore aims at deriving from a limited set of detailed stack flow simulations effective nonlinear impedance laws-both acoustic and thermal. These can then be used to accurately represent the stack in a pure quasi-one-dimensional simulation of the entire system behavior. 


\section{The multiple pressure variable, multiple length scales acoustic solver}

One of the authors has recently [4] extended a Godunov-type compressible flow solver for reliable and accurate operation in the low and zero Mach number regime. The scheme has been designed on the basis of a new multiple length scales asymptotic analysis for low-Mach-number flows. The most important aspect of the analysis, as well as of the associated computational scheme, is that it explicitly deals with low-Mach-number long-wave acoustics, superimposed over a quasi-incompressible small-scale flow including large amplitude density variations. In particular, in the power law expansion of the pressure field in terms of the small Mach number, there appears a first order term $M p^{(1)}$, which depends on the acoustic-scale spatial coordinate only and represents the long-wave acoustic signals. The consequence is that the flow velocities induced by the acoustics are comparable to the those of the underlying small-scale velocity field; this feature distinguishes the analysis from earlier work in the field of low- and zero-Mach-number flow theory.

The numerical scheme used in the present study is derived from the analysis mentioned above. Specifically, it solves the compressible Euler equations

$$
\begin{gathered}
\rho_{t}+c(\rho u)_{x}=0 \\
(\rho u)_{t}+\left(\rho u^{2}+\frac{1}{M^{2}} p\right)_{x}=0 \\
(\rho e)_{t}+\quad(u[\rho e+p])_{x}=0
\end{gathered}
$$

with $(\rho, u, e, p)$ the density, velocity, total energy per unit mass and the pressure, by an operatorsplitting technique involving the following two steps:

$$
\begin{gathered}
\text { System I : } \\
\rho_{t}+\quad(u \rho)_{x}=0 \\
m_{t}+(u m+p)_{x}=0,
\end{gathered}
$$

where $m=\rho u$ and

$$
\begin{array}{ccc}
\text { System II : } & \\
m_{t}+\left(1 / M^{2}-1\right) p_{x} & =0 \\
e_{t}+ & (H m)_{x} & =
\end{array}
$$

where

$$
p=(\gamma-1)\left(e-M^{2} \frac{m^{2}}{2 \rho}\right) \quad \text { and } \quad H=\frac{e+p}{\rho} .
$$

The first step describes the advection of mass and momentum and their changes due to flow divergence. In the non-dimensional notation used here, which is adapted from [4], there appears a factor of $1 / M^{2}$ in the full flow equations from (1). Therefore the pressure gradient in (2) just represents a small perturbation to the advection/compression effects from $(\rho u)_{x}$. This additional term is introduced here as a regularization, which is needed because the system without the pressure term is hyperbolic with double signal speed $u$ but lacking two independent right-eigenvectors. The small perturbation induced in (2) is then accounted for in (3) by including $\left(1 / M^{2}-1\right) p_{x}$ instead of $1 / M^{2} p_{x}$ in the momentum balance. The examples shown in the results section deal with flows in closed tubes. We use standard reflecting wall boundary conditions in this first step to guarantee that there is no convective flux across the rigid ends of these walls. 
The function of the second-split system is two-fold. For the limit of zero Mach number, the analysis in [4] shows that the energy equation reduces to the zero divergence constraint for incompressible flows. The momentum equation then includes the gradient of the second order pressure $p^{(2)}$, which is reponsible for guaranteeing compliance with the divergence contraint. For any small, but non-zero Mach number one finds that the system (3) is hyprbolic with signal speeds (in nondimensional notation) $\pm c / M+O(1)$. Obviously, this system describes fast sound wave propagation in that case. For an accurate numerical description, both the acoustic and the divergence constraint aspects of this equation system need to be carefully accounted for. Guided by the asymptotics one proceeds as follows:

1. Through averaging over $1 / \mathrm{M}$ grid points one obtains the long wave length components of the pressure variations and of the veolocity field.

2. Based on the resulting long wave data, which are interpreted as long wave acoustic initial data for the current time step, one performs a linear acoustic prediction, yielding acoustics-induced changes of the first order pressure $\left(\mathrm{M} \delta p^{(1)}\right)$ and preliminary long-wave momentum updates.

3. Based on the latter predictions, one enters a fully-implicit discretization of system (3) aiming at computing small $O\left(M^{2}\right)$ pressure corrections as well as a divergence-corrected velocity field.

The key features of this procedure are the long wave averaging/filtering, a large time-step method [5] to actually carry out the long-wave acoustic prediction with a time step governed by the convection Courant number and the fact that we compute explicitly separate pressure updates scaled by $M$ and $M^{2}$, corresponding to the pressure expansion $p=p^{(0)}+M p^{(1)}+M^{2} p^{(2)}+\cdots$. It is shown in [7] that such a multiple-pressure procedure is necessary for a compressible flow to remain viable in the zero mach number limit (thereby turning into an incompressible one. For more details, including a discussion of weakly nonlinear acoustic effects and their numerical representation, see [4, 7].)

The key boundary condition in this step is that for the second order pressure $p^{(2)}$. We adjust the pressure gradient at the wall surfaces such that the velocity stays zero there. This guarantees zero energy flux across as well as a suitable constraint on the momenta in the cells adjacent to the walls.

In the simulation of the thermo-acoustic refrigeration system shown in Fig. 1, it is necessary to model the influence of the stack of plates on the acoustic field as well as the energy input that drives the standing acoustic wave and maintains its amplitude. Both these effects appear as sources of momentum and energy for the gas flow; these are implemented in a straightforward fashion in the implicit pressure-correction step (3). The particular functional form of these source terms will be discussed in the following section, and in Section 6 .

\section{Stack flow simulations}

The one-dimensional scheme described in the previous section allows us to accurately simulate the resonant acoustic pulse in the tube including global heat and momentum exchange with the stack and weakly-nonlinear effects. In order to construct a scheme which accurately describes the entire system, we need to first obtain detailed insight into small-scale multi-dimensional flow phenomena around the stack, and then exploit this insight to construct suitable global representations of the stack behavior.

The idea is to perform locally a full-fledged two- or three-dimensional simulation for the vicinity of the plates [9], while relying on the flow becoming quasi-one-dimensional to a good approximation 
away from the stack. Thus, we need to couple the one-dimensional nonlinear acoustic "outer" computation with a subscale multi-dimensional computation in an "inner" region near the plates.

The gasdynamic code handles momentum and energy sources by design, so that the information transferred from the inner to the outer computation involves integrating the global flow forces on the plates as well as the heat transfer to the plates in the inner computation. This provides the effects of the plates on the outer acoustic flow.

The acoustic scale computation yields average mass fluxes through the stack and the acousticsinduced pressure changes, which are responsible for global density and temperature variations in the inner flow. Remember that these acoustically-induced temperature changes are the essential feature that drives thermoacoustic energy transport along the plates. Hence, even though they are small, of order $O(M)$ away from the plates, they must be accurately accounted for.

The multi-dimensional simulation is based on a zero-Mach-number [6], compressible flow model which takes advantage of the fact that the stack is much shorter than the length of the resonance tube. Thus, acoustic wave propagation within the stack is ignored, leading to a simplified flow model. We rely on the Helmholtz decomposition of the velocity vector into irrotational and divergence-free parts, and express the corresponding governing equation in non-dimensional form as:

$$
\begin{gathered}
\mathbf{u}=\nabla \phi+\nabla \times \psi \\
\nabla^{2} \phi=-\frac{1}{\gamma P} \frac{d P}{d t} \\
\frac{\partial \boldsymbol{\omega}}{\partial t}+\nabla \times(\boldsymbol{\omega} \times \mathbf{u})=-\frac{\nabla \rho}{\rho} \times \frac{D \mathbf{u}}{D t}+\frac{1}{\rho R e_{\delta}} \nabla^{2} \boldsymbol{\omega} \\
\nabla^{2} \psi=-\boldsymbol{\omega} \\
\rho \frac{D T}{D t}-\frac{\gamma-1}{\gamma} \frac{d P}{d t}=\frac{1}{P e} \nabla^{2} T
\end{gathered}
$$

where $\mathbf{u}$ is the velocity, $\phi$ is the potential, $\psi$ is the streamfunction, $\boldsymbol{\omega}$ is the vorticity, $\rho$ is the density, $P$ is the thermodynamic pressure, and $T$ is the temperature.

Quantities are normalized with respect to the plate centerline separation distance $\tilde{H}$, angular frequency of the standing wave $\tilde{\Omega}$, mean pressure $\tilde{P}_{0}$ and mean temperature $\tilde{T}_{0}$, where $\sim$ denotes dimensional quantities. This leads to the definition of six dimensionless groups which characterize the flow dynamics, position and shape of the stack: a) The kinematic Reynolds number $\operatorname{Re}_{\delta}=\frac{\tilde{\Omega} \tilde{H}^{2}}{\tilde{\nu}}$ b) the particle displacement parameter $R_{p}=\frac{\tilde{u}_{a}}{\tilde{\Omega} \tilde{H}}$ c) the acoustic amplitude Reynolds number $\left.R e_{a}=\frac{2 \tilde{u}_{a}}{\sqrt{\tilde{\nu} \tilde{\Omega}}} \mathrm{d}\right)$ the Peclet number $P e=\frac{\tilde{\Omega} \tilde{H}^{2}}{\tilde{\alpha}}$ e) the blockage ratio $\frac{\tilde{h}}{\tilde{H}} \mathrm{f}$ ) the plate length parameter $\frac{\tilde{L}}{\tilde{H}}$ and $\mathrm{g}$ ) the dimensionless wave number $k x=\frac{\tilde{\Omega} \tilde{x}}{\tilde{c}}, \tilde{x}$ being the location of the stack from the rigid end of the tube, $\tilde{u}_{a}$ the velocity amplitude of the acoustic wave at the stack position, $\tilde{\nu}$ the kinematic viscosity of the working fluid, $\tilde{h}$ the gap between the plates, and $\tilde{L}$ the length of the stack plates.

To assess the response of the stack to low driving amplitudes where substantial temperature differences are not expected, the Peclet number is set to infinity, i.e., an adiabatic flow model is used. The resulting equations are simulated using a finite difference approach. Briefly, all the spatial derivatives are approximated using second-order centered-differences and the third-order 
Adams-Bashforth scheme is used to update the solution in a fully explicit manner. The flow is assumed irrotational away from the stack plates in the far field. Hence, the impact of the acoustic field is represented by oscillating velocity boundary conditions, i.e., Neumann conditions on the potential.

The Poisson equation for the streamfunction (Eq. 8) needs to be solved at every time step and forms the main overhead in the computational load. To reduce this overhead and memory requirements, a domain decomposition technique is developed and implemented. The computational domain is divided into three rectangular parts; thus the original discrete elliptic system is transformed into three linear systems of equations which are coupled at the interfaces of the domains. A boundary Green's function technique is used to decouple these systems and to enable easy implementaion of fast Fourier solvers.

\section{Results}

\subsection{Nonlinear thermally-driven acoustic resonance}

We have tested the capability of the quasi-one-dimensional code to produce steady state resonant standing waves including nonlinear acoustic effects. To drive the acoustics, we mimic heat addition from the walls of the tube. The idea is as follows: Intially, the gas is at rest and over the length of the left third of the tube the gas temperature has a linear variation with the temperature maximum near the tube end. Next we assume that the tube walls have the same temperature and that their heat capacity is large enough to maintain this temperature distribution even when the gas begins to move. We have discussed how each mass particle traces out a certain temperature gradient in its vicinity due to its combined motion and dilatation under the infuence of the standing wave.

Now consider a mass element next to the tube wall. It experiences the maximum pressure/temperature amplitudes of all particles, while due to the presence of the wall it is locked to one place spatially. As a consequence, its "traced out" acoustically-induced temperature gradient is infinite. The opposite is true for a mass element that is located in the middle of the tube. Here we have a pressure/temperature node, while the particle displacement is maximal. Thus in the middle of the tube the acoustically-induced temperature gradient vanishes. In between, any value of this gradient is achieved and in particular, there is a threshold location where the acoustic gradient matches that of the wall/initial temperature.

To the left of this location, the gas temperature is larger than the local wall temperature when the pressure becomes larger than the mean due to acoustic compression, to the right it is the other way round. Now recall Rayleigh's criterion, which states that an acoustic pulse is amplified if heat is added in the phase of over-pressure, while it is damped if heat is extracted. Assuming a simple linear heat exchange model between the gas and the wall, where the net heat exchange is proportional to, but directed against the local temperature difference, the wall heat exchange will dampen the acoustics left of the critical point on the tempreature ramp, while it will drive to the right of it.

For the following computation we have chosen the temperature ramp such that the critical point for the efficiency of acoustic driving is located at one widths of the tube length, i.e., in the middle of the temperature ramp. Figure 3 shows three time histories of the velocity in the center of the tube for computations with heat exchange being (a) active over the whole length of the wall temperature gradient, (b) active over the left half and (c) active only over the right half of the wall temperature distribution. Figure $3 \mathrm{a}$ shows an almost constant amplitude, showing that there is very little amplification. As the right half of the heating section is isolated from the gas, the acoustic wave is strongly damped in accordance with Rayleigh's criterion. The opposite is true 
when the right half of the heating section is active: We observe a continous amplification, which is maintained until weak shock formation. Then, ultimately the dissipation in the shock balances the heat addition in the driver section and a limiting amplitude is achieved.

Next we have added a momentum source representing the stack resistance at $3 / 4$ length of the tube from the left. If that resistance is strong enough, it controls the amplitude of the acoustic wave. Figure 4 shows again velocities near the middle of the tube long times of persistent oscillations. Figure 4a has been obtained without losses and it is a direct continuation of the computation from Fig. 3c. The run in Fig. 4b includes friction losses It is seen that the momentum losses have led to a saturation of the acoustics at roughly half the amplitude of the case without friction. Also the spatial pressure distributions (not shown here) indicate the presence of a dissipating shock for Fig. $4 \mathrm{a}$, while the acoustic flow in the second case is smooth.

\subsection{Stack flow}

The response of the stack flow to a large variety of conditions has been examined in [9]. Here only one illustrative example is presented. Figure 5 shows the evolution of the streamfunction distribution over half a cycle for a stack with $h / H=0.666$ and $L / H=4$. The device is located at $k x=\pi / 2$ and operated at a drive ratio of $1 \%, R e_{\delta}=2132, R_{p}=66.27$ and $R e_{k}=0.717$. The arrows indicate the magnitude and the direction of the mean velocity at the center of the stack. The contour plots are generated one eighth of a cycle apart at normalized times a) $t=32 \pi / 4$, b) $t=33 \pi / 4$, c) $t=34 \pi / 4$ and d) $t=35 \pi / 4$.

Figure $5 \mathrm{a}$ is generated at the end of the fourth cycle at which time the acoustic velocity vanishes. At the top end of the stack, a separation bubble is observed. At the other end of the stack, streamfunction contours show the presence of a pair of concentrated eddies close to the plates and a pair of weaker eddies away from the plates. As the flow is accelerated upwards, the vortical structures located near the bottom edge of the plate are pushed towards the edges of the plates and deformed in the cross-stream direction. At the "entrance of the channel", the streamfunction distribution suggests the formation of a separation zone; a similar process can be observed at the channel exit. When the acoustic velocity reaches its first peak, vortical structures which existed upstream of the plates have almost been destroyed due to impingement against the plate edges. Meanwhile, the seperation bubbles at the entrance and exit of the channel continue to intensify. The recirculation zones on the opposite sides of the channel are not anchored to the plates but slide along the faces of the plates. These travelling recirculation zones are attached to the vortex bubble which has formed between them, and the three vortices propogate as a single structure.

It is quite obvious that the net friction on the plates and the net heat transfer from the gas to the plates will be depend strongly on the motion and evolution of vortical structures such as those shown above, and that the impact of these vortices cannot be simply guessed using a quasi1D flow model. Thus, our current work aims at deriving from detailed parametric studies of the multi-dimensional flow nonlinear effective friction and heat transfer "laws" which account for the complex flow patterns. The resulting relationships can then be used in a quasi-1D simulation to accurately represent the effective forces and energy exchange rates.

\section{Conclusions}

We have introduced numerical techniques aiming at accurate numerical simulations of a simplified thermoacoustic refrigerator. The overall method consists of a quasi-1D computation scheme for weakly-nonlinear non-constant-entropy acoustics, and a multi-dimensional vorticity/streamfunction/ 
potential formulation for detailed simulation of small-scale flow around the stack. We have demonstrated that the $1 \mathrm{D}$ code is capable of representing wave amplification through heat addition similar to what is actually observed in experiments. We have also studied mechanisms that control the acoustic amplitudes. It is shock dissipation in the loss-free case, while the presence of momentum losses leads to a quasi-steady state at lower amplitudes and prevents shock formation.

The computational scheme for simulating the small-scale flow around the stack has been designed to elegantly couple to the outer acoustic computational scheme. Using the average mass flux through the device and the global acoustic pressure variations as input, the code yields the detailed flow evolution around the stack. Integration of all flow forces and heat fluxes yields the feed-back to the outer acoustic flow in the form of effective local source terms of energy and momentum.

Our future work will involve (a) fully coupled simulations for long wave acoustics and the stack flow and (b) derivation of effective nonlinear/nonlocal impedance laws that can be used directly in quasi-1D simulation to obtain an accurate global representation of multi-dimensional phenomena around the stack, and thus avoid the need for computing the multi- dimensional response of the stack at every "outer" time step.

\section{Acknowledgement}

ASW and OMK would like to acknowledge support from the Office of Naval Research. Computations were performed at the Pittsburgh Supercomputer Center. RK thanks the Deutsche Forschungsgemeinschaft for their support in the framework of the Special Research Programme on "Flow Simulation on High-Performance Computers".

\section{References}

[1] Atchley, A., Hofler, T., Muzzerall, M., Kite, M. and Ao, C., "Acoustically Generated Gradients in Short Plates," J. Acoust. Soc. Am., 88 (1), pp. 251, 1990.

[2] Herman, C. and Wetzel, M., "Design of a Thermoacoustic Refrigerator," Winter Annual Meeting, ASME, 1995.

[3] Hofler, T.J., Thermoacoustic Refrigerator Design and Perfomance, Ph.D thesis, Physics Department, University of California at San Diego, 1986.

[4] Klein, R., "Semi-Implicit Extension of a Godunov-Type Compressible Flow Solver Based on Low Mach Number Asymptotics I: One-Dimensional Flow", J. Comp. Phys., to appear, 1995.

[5] LeVeque, R.J., “A Large Time Step Generalization of Godunov's Method for Systems of Conservation Laws," SIAM J. Num. Anal., 22, pp. 1051-1073.

[6] Majda, A. and Sethian, J., "The Derivation and Numerical Solution of the Equations for Zero Mach Number Combustion," Combust. Sci. Tech., 42, pp. 185, 1985.

[7] Munz, C.D. and Klein, R., "Multiple Pressure Variable Extension of Incompressible Flow Solvers to the Weakly Compressible Regime," submitted for publication.

[8] Lord Rayleigh, Theory of Sound, Dover, New York, 1945.

[9] Worlikar, A.S. and O. Knio, O., "Numerical Simulation of a Thermoacoustic Refrigerator. Part I : Unsteady Adiabatic Flow around the Stack," submitted to J. Comp. Phys., June 1995. 
(a)

(b)

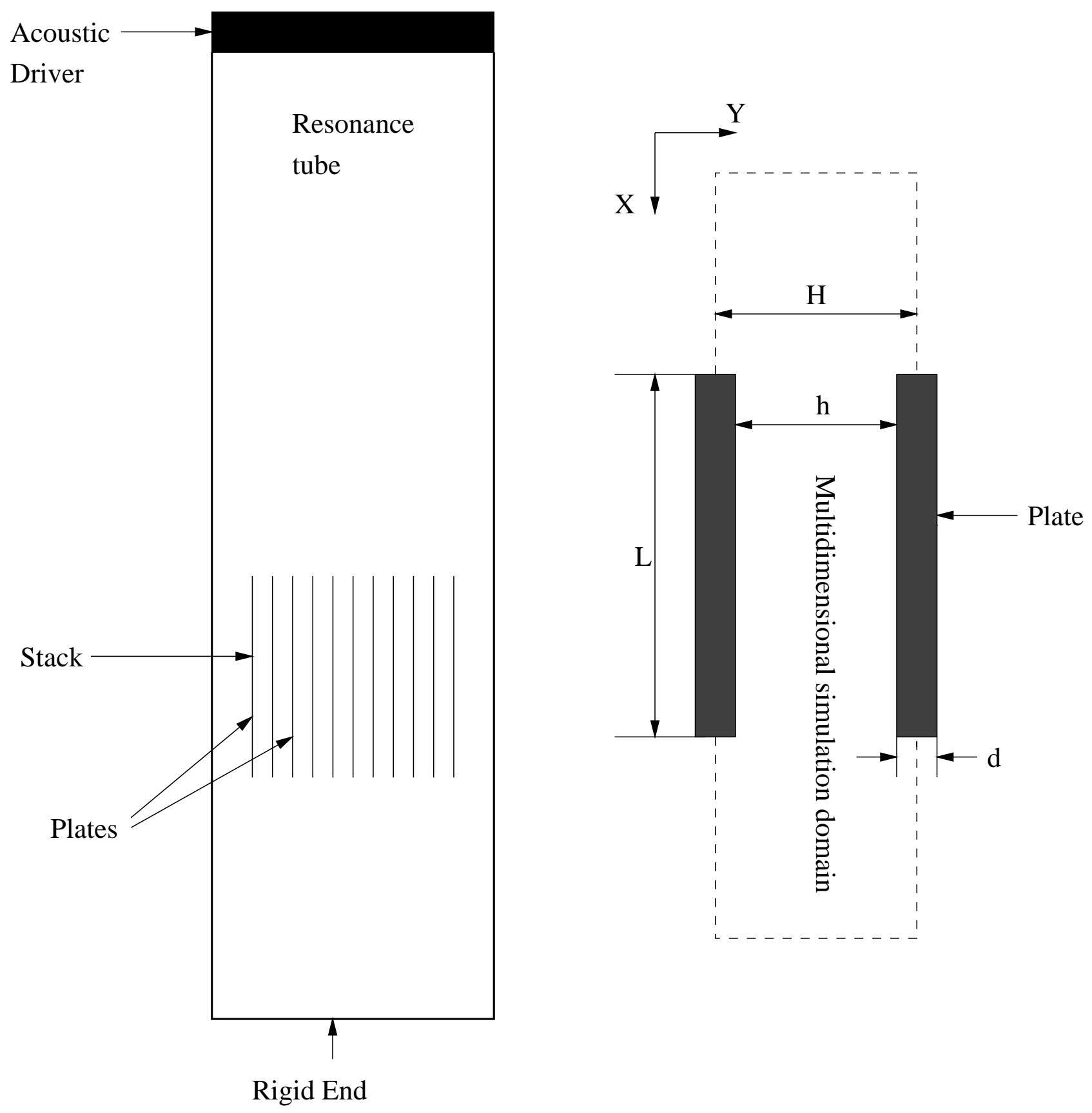

Figure 1.

Figure 1: Schematic illustration of the thermo-acoustic device. 


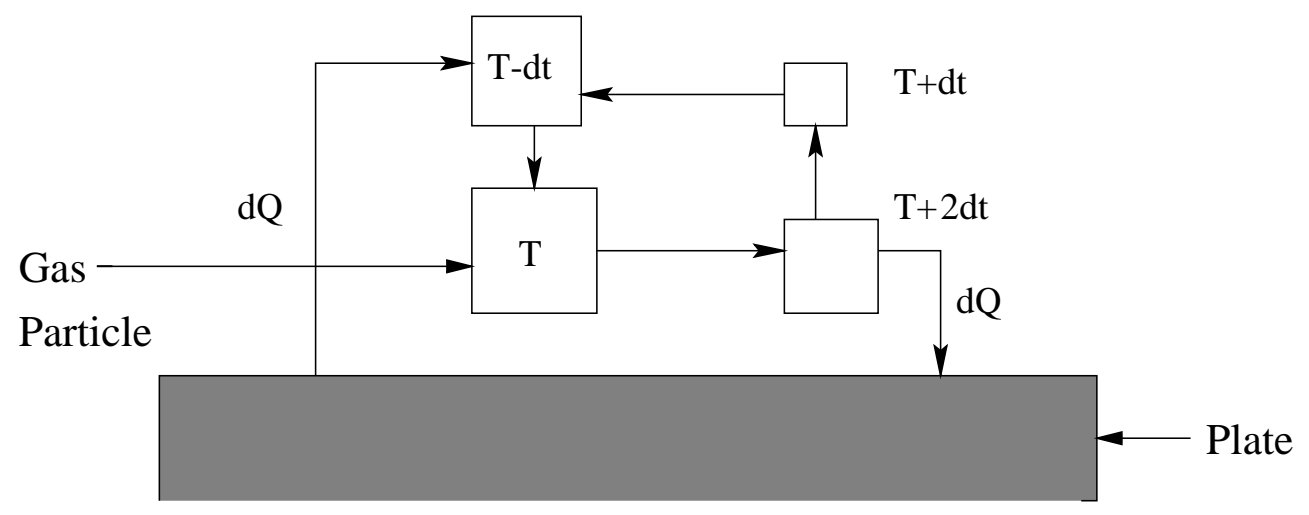

Figure 2.

Figure 2: Schematic illustration of heat exchange mechanism for a gas particle. 
a)

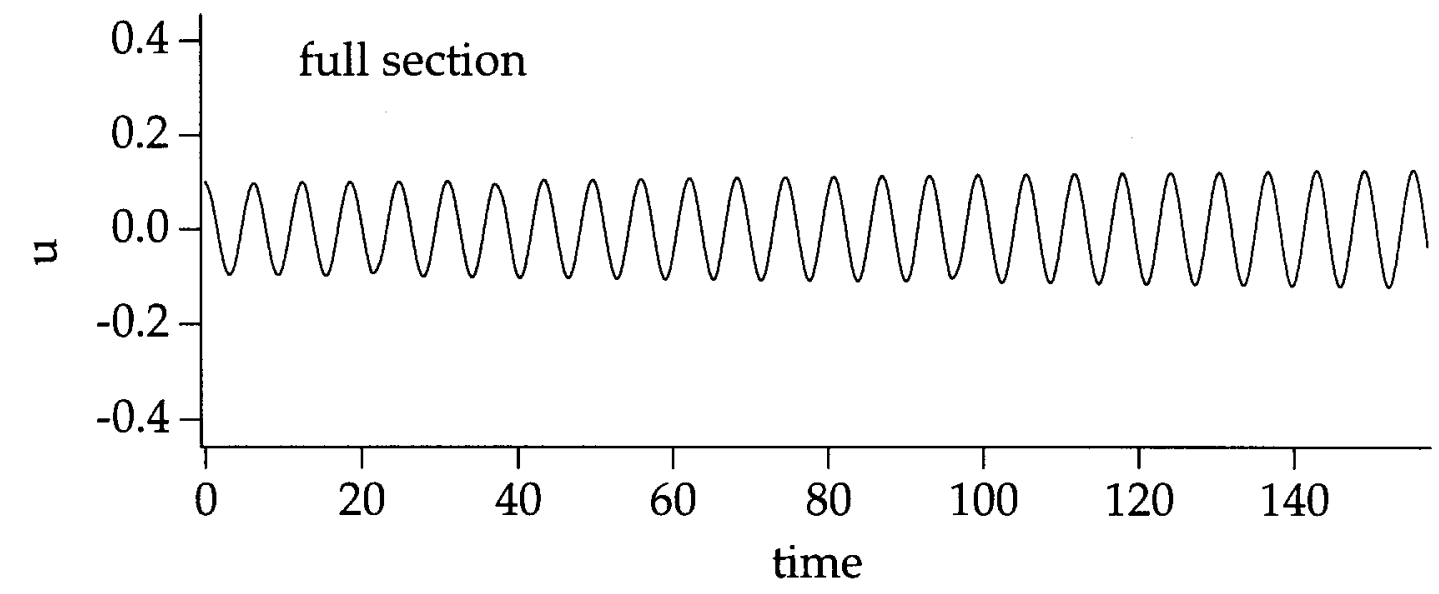

b)

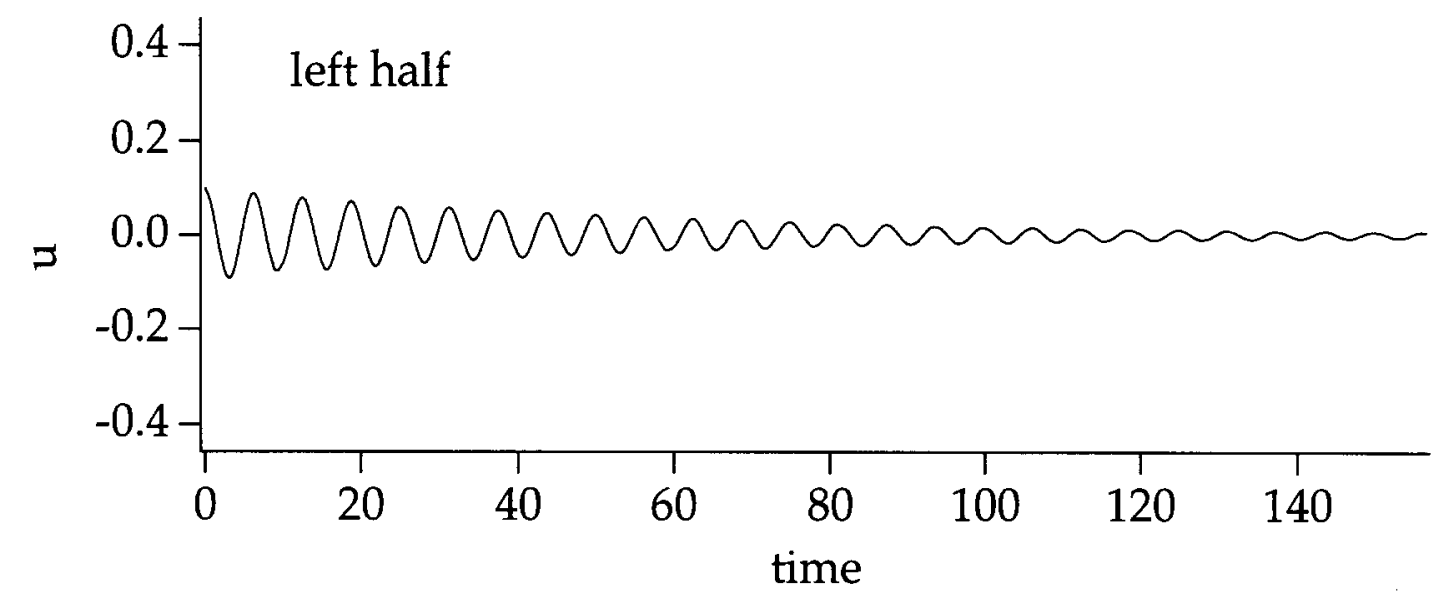

c)

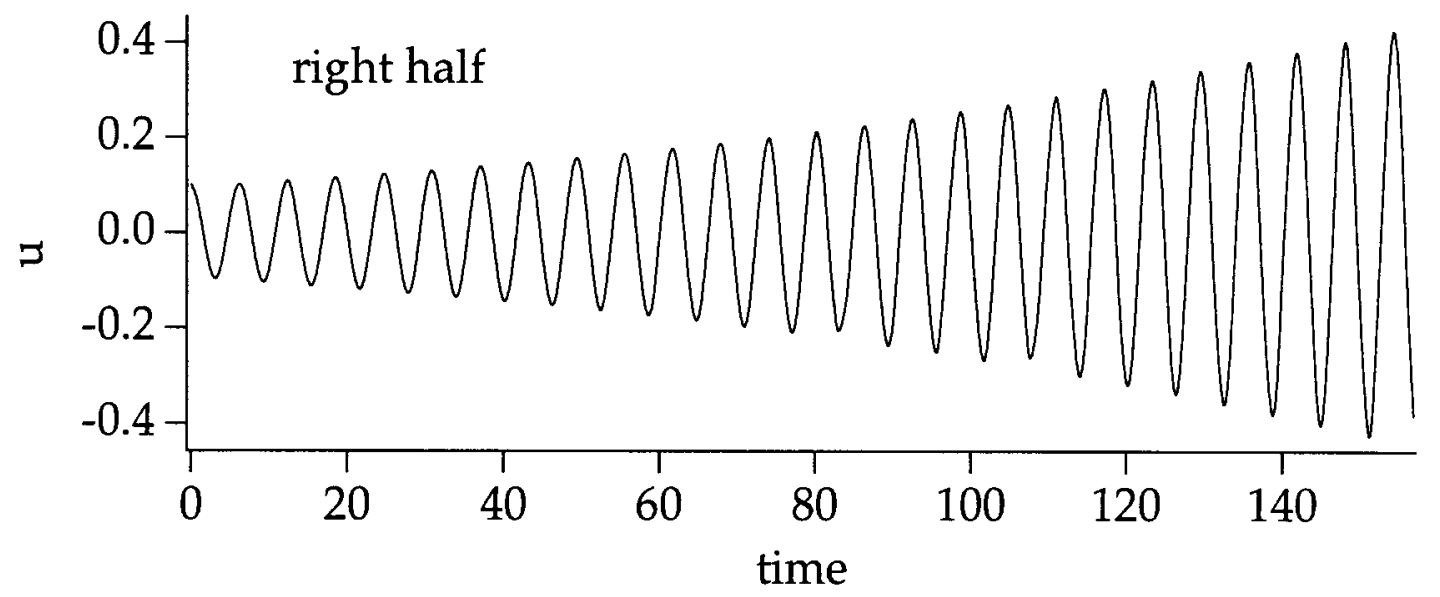

Figure 3: Maximum velocity amplitudes for a nonlinear acoustic standing wave under various driving conditions. Heat addition through a linear temperature ramp over: a) left third of the tube, b) left sixth of tube (left half of total temperature ramp), c) one sixth to two sixth of the tube (right half of temperature ramp). 


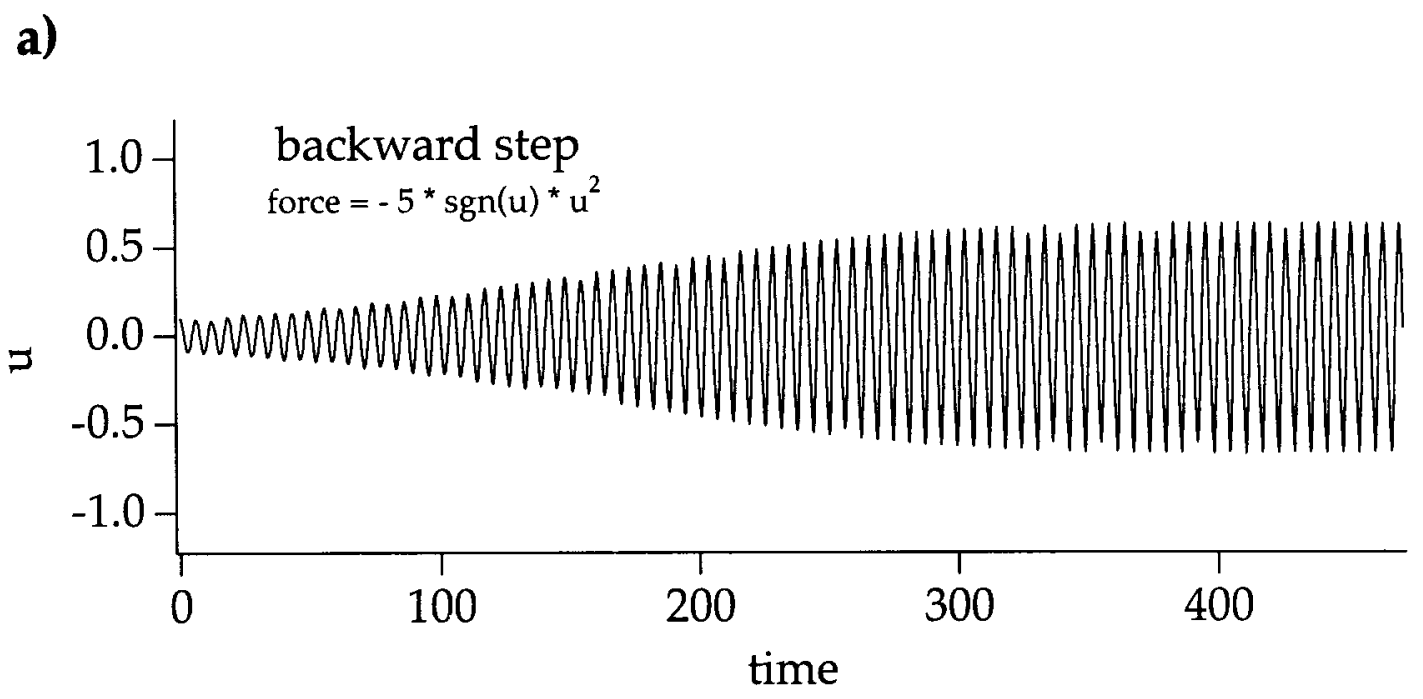

b)

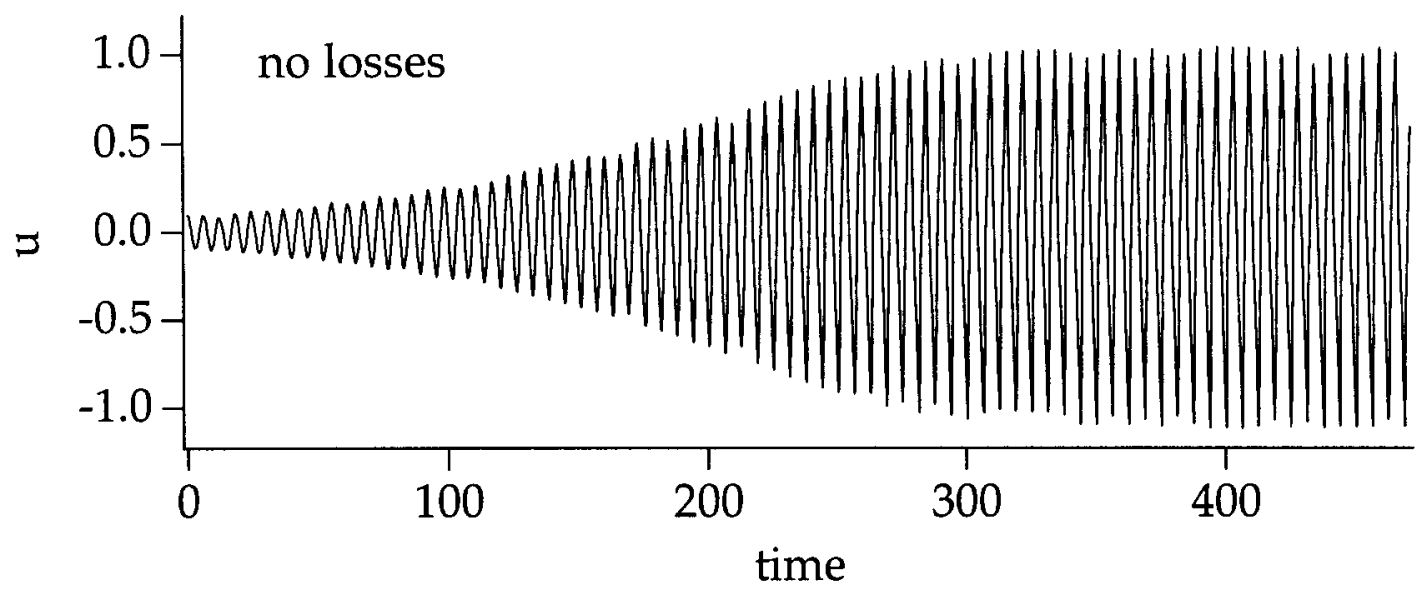

Figure 4: Maximum velocity amplitudes for a nonlinear acoustic standing wave driven as in Fig. 3c, but with different saturation mechanisms. a) no losses; saturation through weak shock formation, b) quadratic friction loss at 3/4 length of the tube from the left, mimicking the effect of a backward facing step in quasisteady flow. 

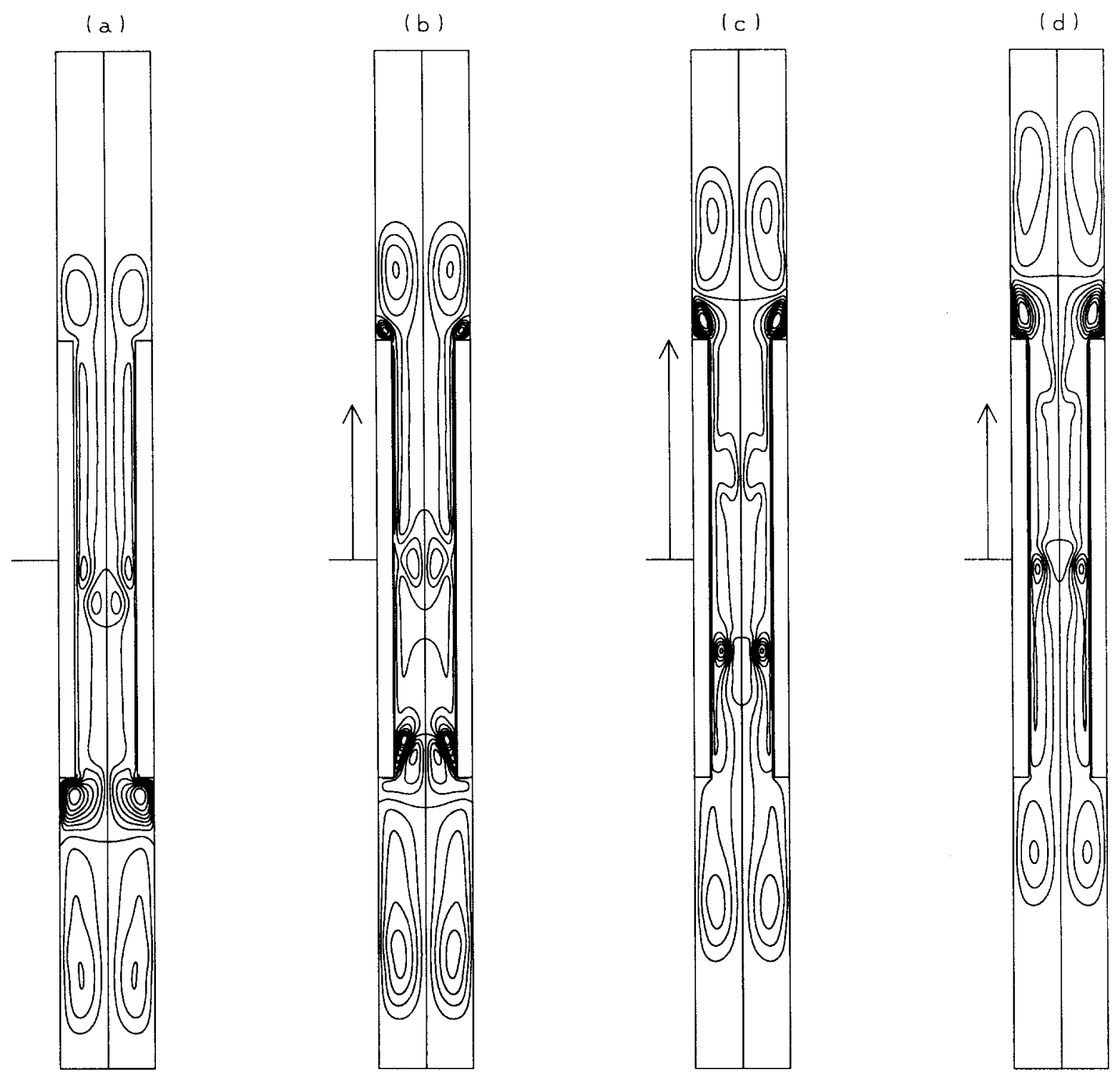

Figure 5: Evolution of the streamfunction distribution over half a cycle for a stack with $\mathrm{h} / \mathrm{H}=$ 0.666 and $\mathrm{L} / \mathrm{H}=4.0$. The device is located at $k x=\pi / 2$ and operated at drive ratio of $1 \%$ and the following parameters: $R e_{\delta}=2132, R_{p}=66.27$ and $R e_{k}=0.717$. The arrows indicate the magnitude and the direction of the mean velocity at the center of the stack. The contour plots are generated one eighth of a cycle apart at normalized times a) $t=32 \pi / 4$, b) $t=33 \pi / 4$, c) $t=34 \pi / 4$, d) $t=35 \pi / 4$. 